Why appreciation of pulmonary vitamin D metabolism should temper expectations for vitamin $D$ supplementation to reduce disease severity and improve clinical outcome of COVID-19

\author{
Rob Janssen ${ }^{1}$, Jona Walk ${ }^{2}$ and Jody M.W. van den Ouweland ${ }^{3}$ \\ ${ }^{1}$ Department of Pulmonary Medicine, Canisius-Wilhelmina Hospital, Nijmegen, The \\ Netherlands; ${ }^{2}$ Department of Internal Medicine, Canisius-Wilhelmina Hospital, Nijmegen, \\ The Netherlands; ${ }^{2}$ Department of Clinical Chemistry, Canisius-Wilhelmina Hospital, \\ Nijmegen, The Netherlands
}

Correspondence: Dr. Rob Janssen, Department of Pulmonary Medicine, Canisius-

Wilhelmina Hospital, Weg door Jonkerbos 100, 6532 SZ Nijmegen, The Netherlands. Email: rob.janssen@cwz.nl

\begin{abstract}
Vitamin D is a nutrient with anti-inflammatory properties whose role is currently being evaluated in COVID-19. Although studies are conflicting, they seem to suggest a role for vitamin $\mathrm{D}$ in reducing disease susceptibility but not in improving clinical outcome. In order to understand why vitamin $\mathrm{D}$ does not seem to have much effect on decreasing disease severity, it is essential to appreciate pulmonary vitamin D metabolism. To reach the pulmonary compartment, vascular endothelial cells would need to take up vitamin D from the blood stream, but they lack vitamin D receptor (VDR) and the activating enzyme CYP27B1. Endothelialitis - an important disease manifestation of COVID-19 - is therefore not expected to be directly affected by vitamin D. Bronchial epithelial cells are usually among the first to be infected with SARS-CoV-2. They do express both VDR and CYP27B1, but circulating vitamin D may not reach bronchial epithelial cells without transportation from the blood stream through the blood vessel wall. Inhalation therapy with vitamin D has therefore been suggested as an alternative for oral administration to bypass endothelial cells and efficaciously target bronchial epithelium. In conclusion, based on the principles of pulmonary vitamin D metabolism, it is not expected that vitamin $\mathrm{D}$ administration has a significant effect on COVID-19 severity. Vitamin D is more likely to reduce SARS-CoV-2 susceptibility, but reaching the airways with oral supplementation will be difficult and vitamin D inhalation therapy should be considered.
\end{abstract}

Keyword: Vitamin D; COVID-19; SARS-CoV-2; endothelialithis; CYP27B1; vitamin D receptor; CYP24A1

\title{
Introduction
}

Vitamin D can be synthesized in the skin as well as obtained from foods and supplements. It has myriad effects throughout the body, ranging from its classical role in bone health and calcium-phosphorus metabolism to modulation of the immune response in communicable and noncommunicable diseases. A meta-analysis conducted before the emergence of SARS-CoV2 demonstrated that oral vitamin $\mathrm{D}$ administration protects against acute respiratory tract infections [1]. It has therefore been speculated that vitamin D may play a favorable role in COVID-19.

\section{Observational studies}

Observational studies assessing effects of vitamin D on COVID-19 susceptibility and severity have been conflicting. In a small Swiss cohort of a little more than a hundred individuals who underwent nasopharyngeal swab PCR analysis for SARS-CoV-2, vitamin D levels were 
significantly lower in those who were positive compared those who were negative [2]. However, in a larger British study of more than half a million biobank participants no significant link was found between vitamin D levels and COVID-19-related mortality after correction for confounders [3]. Panagiotou et al., on the other hand, showed higher frequency of vitamin D deficiency in COVID-19 patients admitted to the intensive care unit but also failed to demonstrate an effect of vitamin D status on survival [4]. Whereas Walk et al. did not find differences in vitamin D status between hospitalized COVID-19 patients with poor and good disease outcomes [5], Jain et al. demonstrated a strong association between vitamin D deficiency and COVID-19-related mortality [6]. Israel et al. performed a large multiethnic population-based study and found a highly significant correlation between prevalence of vitamin D deficiency and incidence of confirmed SARS-CoV-2 infections [7]. Influence of vitamin D status on COVID-19-related hospital admissions, ICU admissions and mortality were not assessed in this study [7].

\section{Intervention studies}

To date, two intervention studies have evaluated the effects of vitamin $\mathrm{D}$ administration in hospitalized COVID-19 patients with again conflicting results $[8,9]$. In a pilot study, highdose vitamin D reduced ICU admission rate among hospitalized COVID-19 patients [8]. However, this study had a small sample size and comorbidities (i.e. diabetes and hypertension) were unevenly distributed between the intervention and control groups, and the differences lost significance after correcting for this. A more extensive clinical trial is currently ongoing at this center. Preliminary data from a larger randomized, double-blind, placebo-controlled multicenter trial in Brazilian hospitalized COVID-19 patients demonstrated no favorable effects of vitamin D on length of hospital stay, ICU admission rate, need for mechanical ventilation or death [9]. The effects of vitamin D3 supplementation were not better in vitamin D deficient $(25(\mathrm{OH}) \mathrm{D}<50 \mathrm{nmol} / \mathrm{L}) \mathrm{COVID}-19$ patients but tended to be even worse than in those who were vitamin D sufficient at baseline [9].

\section{Systemic and pulmonary vitamin D metabolism}

Appreciation of systemic and pulmonary vitamin D metabolism may temper high expectations of vitamin D supplementation to improve COVID-19 outcome. After dermal synthesis or absorption from the gut, vitamin $\mathrm{D}$ has to be converted by hepatocytes into the storage metabolite 25 -hydroxyvitamin $\mathrm{D}(25(\mathrm{OH}) \mathrm{D})$. The hormonal metabolite 1,25 dihydroxyvitamin $\mathrm{D}(1,25(\mathrm{OH}) 2 \mathrm{D})$ is subsequently acquired through conversion of $25(\mathrm{OH}) \mathrm{D}$ by the activating enzyme CYP27B1. Kidneys are the most important contributors to systemic $1,25(\mathrm{OH}) 2 \mathrm{D}$ levels. Active vitamin $\mathrm{D}$, however, can also be locally formed in the lungs from free $25(\mathrm{OH}) \mathrm{D}$. Whereas the vast part of serum $25(\mathrm{OH}) \mathrm{D}$ is bound to vitamin D-binding protein, which can only be taken up by cells that express megalin (mainly renal proximal tubule cells), a small proportion of $25(\mathrm{OH}) \mathrm{D}$ is circulating in the free form. This fraction can also be taken up by megalin-negative cells that express vitamin D receptor (VDR). In lungs, VDR and CYP27B1 are expressed in bronchial epithelium but not in endothelial cells [10]. In contrast, the $1,25(\mathrm{OH}) 2 \mathrm{D}$-inactivating enzyme CYP24A1 is expressed by both pulmonary epithelium and endothelium (Figure 1) [10]. 


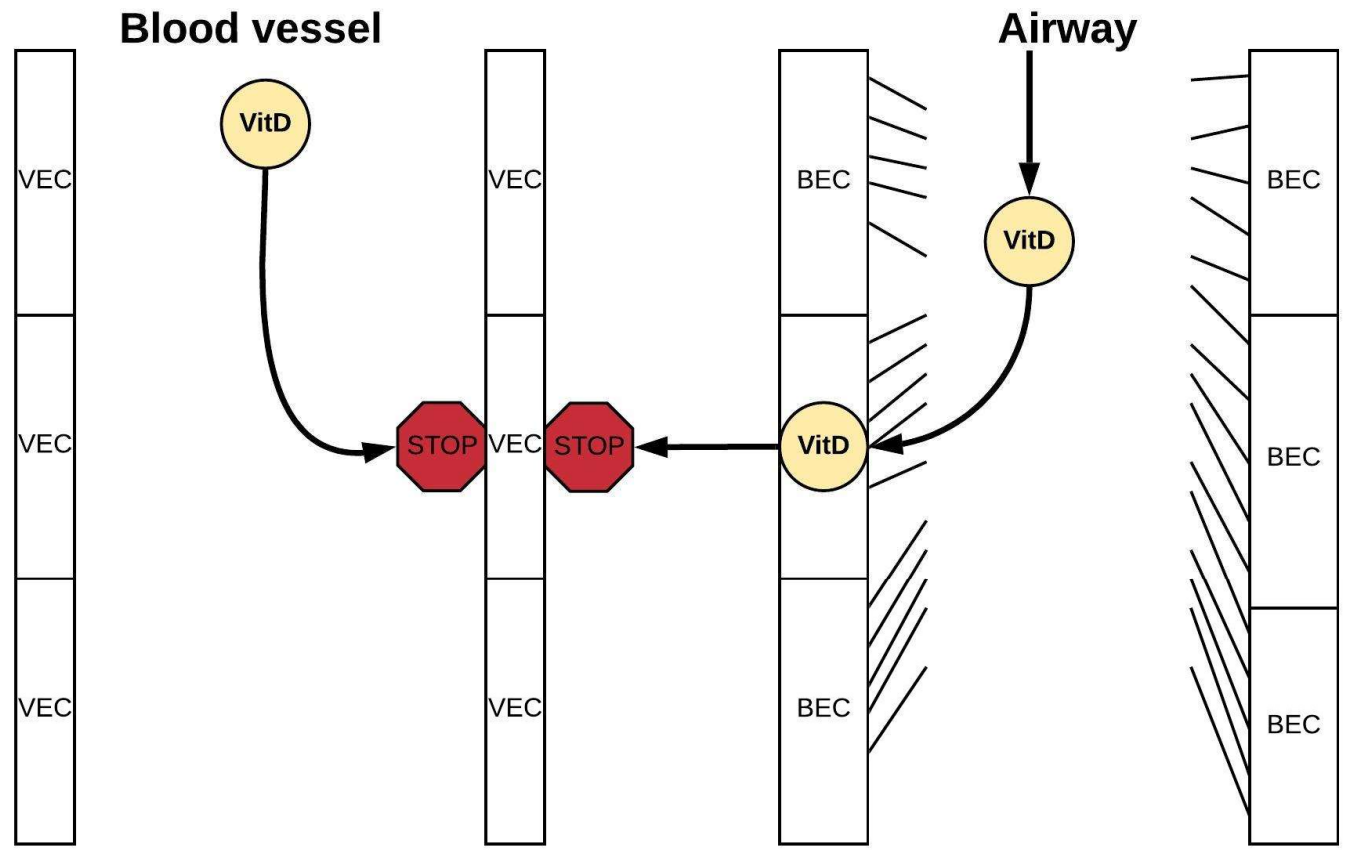

Figure 1. Given that vascular endothelial cells (VEC) lack vitamin D receptor (VDR) and CYP27B1, they are unable to use $25(\mathrm{OH}) \mathrm{D}$ themselves (by converting this storage vitamin D into the active metabolite $1,25(25 \mathrm{OH}) \mathrm{D} 2)$. In contrast, bronchial epithelial cells (BEC) have VDR as well as CYP27B1 and vitamin D could therefore have favorable effects in these cells. However, vitamin D delivery through VEC is not possible, because they lack VDR. Vitamin $\mathrm{D}$ inhalation therapy bypasses VEC and directly delivers vitamin D to BEC.

\section{Airway epithelium}

Epithelial cells that cover upper and lower airways are usually the first cells to be infected by SARS-CoV-2. Vitamin D may play a favorable role in these infected epithelial cells by dampening potentially devastating hyperinflammatory responses induced by the virus. Delivery of free $25(\mathrm{OH}) \mathrm{D}$ from the blood stream to bronchial epithelium is problematic given the inability of passage through pulmonary endothelial cells that lack VDR (Figure 1) [10]. Vitamin D inhalation therapy has therefore been proposed as an alternative for oral administration to effectively reach bronchial epithelial cells [10]. Alternatively, vitamin D droplets - rather than tablets - might be used to bypass the endothelium. Israel et al. demonstrated a protective effect from liquid vitamin $\mathrm{D}$ formulations against SARS-CoV-2 infections (OR 0.9) in a large population-based study, whereas use of vitamin D tablets was associated with increased risk (OR 1.2) [7]. A possible explanation might be that a significant proportion of vitamin D droplets is probably directly absorbed by the epithelial cells of the oropharynx and does not have to pass endothelial cells.

\section{Endothelialitis}

Vascular endothelial cells seem to play a more important role in the pathogenesis of COVID19 than of other respiratory tract infections. Data from postmortem lung examinations suggest that endothelialitis is a more frequent contributor to mortality in patients infected with SARSCoV-2 than with influenza A [11]. Absent VDR and CYP27B1 expression combined with presence of CYP24A1 in pulmonary endothelial cells, however, prevent vitamin D supplementation from having a direct favorable impact on endothelialitis [10].Vitamin D may 
have some indirect effect on endothelialitis through modulation of immune cells, which stained occasionally but weakly positive for VDR and inconclusively for CYP27B1 [10].

\section{Elastic fiber metabolism and vitamin $\mathrm{K}$}

Elastic fibers are crucial components of the extracellular matrix in dynamic tissues such as lungs and blood vessels to facilitate deformability and resilience. Their integrity and function may be hampered by proteolytic degradation as well as calcification. Partially degraded elastic fibers are particularly vulnerable to calcium because of their increased polarity [12]. Elastic fiber mineralization, on the other hand, may stimulate elastolysis as synthesis of proteases is upregulated in parallel to increasing calcium content [13]. It has been demonstrated that levels of both proteases and markers of elastic fiber degradation are increased in COVID-19 patients and related to poor prognosis [14,15]. Matrix Gla protein (MGP) is a vitamin K-dependent calcification inhibitor that is small enough to protect the interior of elastic fibers [16-18]. It has been demonstrated that vitamin $\mathrm{K}$ deficiency is ubiquitous in hospitalized COVID-19 patients and related to poor disease outcomes as well as accelerated elastic fiber degradation [15]. Vitamin D supplementation may be particularly troublesome in a state of vitamin K deficiency [19], as vitamin D is thought to further decrease vitamin K status and stimulate elastolysis [18,20,21]. It may therefore be prudent to combine any vitamin D supplementation in COVID-19 with $\mathrm{K}$ in order to prevent these negative effects.

\section{Concluding remarks}

Data from observational and intervention studies on the effect of vitamin D status and vitamin D administration during COVID-19 are conflicting. In general, larger studies of higher quality demonstrate effects on disease susceptibility [7] but not on severity or disease outcome [3,9]. Vitamin D supplementation is generally considered to be safe [22], however, this may not always be the case [19]. Despite the lack of data demonstrating efficacy and safety, some governments actively advise their citizens to use vitamin D supplementation. Based on the above, we find these recommendations premature and propose reconsideration. We argue that intervention studies should assess the combined effects of vitamin D and K on COVID-19 disease severity rather than of vitamin D monotherapy.

\section{References}

1. Adrian R Martineau, David A Jolliffe, Lauren Greenberg, et al. Vitamin D supplementation to prevent acute respiratory infections: individual participant data metaanalysis. Health Technol Assess 2019;23(2):1-44.

2. Antonio D'Avolio, Valeria Avataneo, Alessandra Manca, et al. 25-Hydroxyvitamin D Concentrations Are Lower in Patients with Positive PCR for SARS-CoV-2. Nutrients 2020;12(5):1359.

3. Claire E Hastie, Jill P Pell, Naveed Sattar. Vitamin D and COVID-19 infection and mortality in UK Biobank. Eur J Nutr 2020;1-4.

4. Grigorios Panagiotou, Su Ann Tee, Yasir Ihsan, et al. Low serum 25-hydroxyvitamin $\mathrm{D}(25[\mathrm{OH}] \mathrm{D})$ levels in patients hospitalized with COVID-19 are associated with greater disease severity. Clin Endocrinol (Oxf). 2020 Jul 3; Online ahead of print.

5. Jona Walk, Anton S.M. Dofferhoff, Jody M.W. van den Ouweland, Henny van Daal, Rob Janssen. Vitamin D - contrary to vitamin K - does not associate with clinical outcome in hospitalized COVID-19 patients. MedRxiv 2020.11.07.20227512; doi: https://doi.org/10.1101/2020.11.07.20227512. 
6. Anshul Jain, Rachna Chaurasia, Narendra Singh Sengar, et al. Analysis of vitamin D level among asymptomatic and critically ill COVID-19 patients and its correlation with inflammatory markers. Sci Rep. 2020;10(1):20191.

7. Ariel Israel, Assi Albert Cicurel, Ilan Feldhamer, et al. The link between vitamin D deficiency and Covid-19 in a large population.

MedRxiv 2020.09.04.20188268; doi: https://doi.org/10.1101/2020.09.04.20188268

8. Marta Entrenas Castillo, Luis Manuel Entrenas Costa, José Manuel Vaquero Barrios, et al. Effect of calcifediol treatment and best available therapy versus best available therapy on intensive care unit admission and mortality among patients hospitalized for COVID-19: A pilot randomized clinical study. J Steroid Biochem Mol Biol. 2020;203:105751.

9. Igor H. Murai, Alan L. Fernandes, Lucas P. Sales, et al. Effect of Vitamin $\mathrm{D}_{3}$ Supplementation vs Placebo on Hospital Length of Stay in Patients with Severe COVID19: A Multicenter, Double-blind, Randomized Controlled Trial. MedRxiv 2020.11.16.20232397; doi: https://doi.org/10.1101/2020.11.16.20232397

10. Carolien Mathyssen, Celine Aelbrecht, Jef Serré, et al. Local expression profiles of vitamin D-related genes in airways of COPD patients. Respir Res. 2020;21(1):137.

11. Maximilian Ackermann, Stijn E Verleden, Mark Kuehnel, et al. Pulmonary Vascular Endothelialitis, Thrombosis, and Angiogenesis in Covid-19. N Engl J Med. 2020;383(2):120128.

12. Federica Boraldi, Pasquale Moscarelli, Francesco Demetrio Lofaro, Carla Sabia ${ }^{1}$, Daniela Quaglino. The mineralization process of insoluble elastin fibrillar structures: Ionic environment vs degradation. Int J Biol Macromol. 2020;149:693-706.

13. Jeoung Soo Lee, Dina M Basalyga, Agneta Simionescu, Jason C Isenburg, Dan T Simionescu, Narendra R Vyavahare. Elastin calcification in the rat subdermal model is accompanied by up-regulation of degradative and osteogenic cellular responses. $\mathrm{J}$ Pathol. 2006 ;168(2):490-8.

14. T Ueland, J C Holter, A R Holten, et al. Distinct and early increase in circulating MMP-9 in COVID-19 patients with respiratory failure. J Infect. 2020;81(3):e41-e43.

15. Anton S M Dofferhoff, Ianthe Piscaer, Leon J Schurgers, et al. Reduced vitamin K status as a potentially modifiable risk factor of severe COVID-19. Clin Infect Dis. 2020 Aug 27; Online ahead of print.

16. G Luo, P Ducy, M D McKee, et al. Spontaneous calcification of arteries and cartilage in mice lacking matrix GLA protein. Nature. 1997;386(6620):78-81.

17. Paul A Price, Damon Toroian, Joo Eun Lim. Mineralization by inhibitor exclusion: the calcification of collagen with fetuin. J Biol Chem. 2009;284(25):17092-101.

18. P A Price, J R Buckley, M K Williamson. The amino bisphosphonate ibandronate prevents vitamin $\mathrm{D}$ toxicity and inhibits vitamin $\mathrm{D}$-induced calcification of arteries, cartilage, lungs and kidneys in rats. J Nutr. 2001;131(11):2910-5.

19. Adriana J van Ballegooijen, Joline W J Beulens, Charlotte A Keyzer, et al. Joint association of vitamins D and K status with long-term outcomes in stable kidney transplant recipients. Nephrol Dial Transplant. 2020;35(4):706-714.

20. Adriana J van Ballegooijen, Joline W J Beulens, Leon J Schurgers, et al. Effect of 6Month Vitamin D Supplementation on Plasma Matrix Gla Protein in Older Adults. Nutrients. 2019;11(2):231.

21. N Niederhoffer, Y V Bobryshev, I Lartaud-Idjouadiene, P Giummelly, J Atkinson. Aortic calcification produced by vitamin D3 plus nicotine. J Vasc Res. 1997;34(5):386-98. 22. Fiona Mitchell. Vitamin-D and COVID-19: do deficient risk a poorer outcome? Lancet Diabetes Endocrinol. 2020;8(7):570. 